25 th SOFT, 15 - 19 September 2008, Rostock, Id. Nr. 367

First author: Louis Giannone, Boltzmannstraße 2, D-85748 Garching, Germany

email: Louis.Giannone@ipp.mpg.de; telephone: +49 893299 1499, fax:+49 893299961499

\title{
Real time magnetic field and flux measurements for tokamak control using a multi-core PCI Express system
}

L.Giannone, W.Schneider, P.J.McCarthy, A.C.C.Sips, W.Treutterer, K.Behler, T.Eich, J.C.Fuchs, N.Hicks, A.Kallenbach, M.Maraschek, A.Mlynek, G.Neu, G.Pautasso, G.Raupp, M.Reich, K.H.Schuhbeck, J.Stober, F.Volpe, T. Zehetbauer and ASDEX Upgrade Team Max-Planck-Institut für Plasmaphysik, IPP-EURATOM Association, 85748 Garching, Germany

M.Cerna, T.Debelle, B.Marker, S,McCaslin, M.Munroe and L.Wenzel, National Instruments Corporation, Austin, TX 78759-3504, USA

\begin{abstract}
The existing real time system for the position and shape control in ASDEX Upgrade has been extended to calculate magnetic flux surfaces in real time using a multi-core PCI Express system running LabVIEW RT. The availability of reflective memory for LabVIEW RT will allow this system to be connected to the control system and other diagnostics in a multiplatform real time network. The measured response of each magnetic probe to the individual poloidal field coil currents in the absence of plasma current is compared to the calculated value. Prior to a tokamak discharge this comparison can be used to check for failure of the magnetic probe, flux loop or integrator.
\end{abstract}

\section{Introduction}

The need for locating the $\mathrm{q}=2$ or $\mathrm{q}=3 / 2$ rational magnetic flux surface position in real time for NTM stabilisation experiments in ASDEX Upgrade [1] has driven the development of a dedicated data acquisition system to achieve this goal. Measurements of integrated magnetic field from magnetic probes and flux loops and measurements of the currents flowing in the poloidal and toroidal field coils of ASDEX Upgrade make up the set of 100 measurements 
used as inputs to the CLISTE tokamak equilibrium code. This code reconstructs the magnetic flux surfaces in ASDEX Upgrade [2] and cannot be presently carried out in real time.

For the real time control system, a function parameterisation algorithm is used to calculate the magnetic equilibrium [3]. This statistical analysis of randomly chosen equilibrium states generates 2 sets of matrix coefficients. The calculation of 95 equilibrium shape and position parameters and the magnetic flux surfaces on a 39x69 grid is reduced to the product of these matrices and a vector of 231 elements consisting of a second degree polynomial in the leading 20 principal components of a vector of 60 measurements. For NTM stabilisation, the mirror has to be set to the angle required to deposit Electron Cyclotron Current Drive (ECCD) power on the rational flux surface. The angle required for the mirror to achieve this is a function of the spatial co-ordinates of the rational flux surface. The spatial co-ordinates of the rational flux surface therefore need to be communicated in real time to the control system by the dedicated data acquisition system [4].

\section{Data acquisition and real time communication}

The data acquisition system for the magnetic field and flux measurements currently used by the VxWorks real time control system system of ASDEX Upgrade consists of a compact PCI unit with 96 channel $250 \mathrm{kHz} 16$ bit D-TACQ digitisers [5]. These digitisers are connected with an optical fibre PCI-PCI bridge to the control system. The lack of multi-core support for VxWorks at the beginning of the project was the basis of the decision to build a new dedicated data acquisition system for calculating flux surfaces in real time. The availability of multicore support for LabVIEW RT and good results achieved in preliminary tests with minimal development time led to the final choice of LabVIEW RT 8.6 as the operating system. An overview of the new data acquisition system for real time magnetic flux surfaces and the VxWorks control system of ASDEX Upgrade is shown in Figure 1. The new data acquisition 
system is based on a Tyan i5000VF motherboard with 2 PCI Express slots, 2 PCI slots, a PCIX slot, 2 GB FB-DIMM memory and a Gigabit Ethernet port [6]. The new data acquisition system consists of 80 analogue inputs recorded simultaneously by 10 PXI 6143 S Series cards with 16 bit ADC's and a sampling rate of $10 \mathrm{kHz}$ for the duration of the $20 \mathrm{~s}$ discharge. The motherboard has sockets for two quad core Intel Xeon X5365 3.0 GHz processors and is connected to the data acquisition cards in a 18 slot PXI chassis via a PCI Express-PXI bridge. This bridge can connect to 2 PXI chassis and transfer data at rates up to $80 \mathrm{MByte} / \mathrm{s}$ per chassis. With two available PCI Express slots, acquisition of up to 512 channels from 4 chassis is possible. A third party 64 bit synchronisation card connected to the central timer by optical fibre occupies a PCI slot. It generates time stamps and a synchronous $10 \mathrm{MHz}$ clock for the PXI chassis.

Real time measurement and control systems using text based programming of VxWorks and real time Linux operating systems $[4,7-12]$ are commonly used in the fusion community. The chosen LabVIEW RT platform uses the graphical programming based data flow paradigm of LabVIEW to realise the multi-threaded real time data acquisition application. With a large library of examples and signal processing algorithms, a user friendly development environment and a wide range of available hardware including FPGA's, it is also possible to build a variety of LabVIEW RT based plasma diagnostics [13-15]. The separation of data acquisition cards and computer facilitates an easy migration path for upgrading the motherboard when new CPU's become available. 


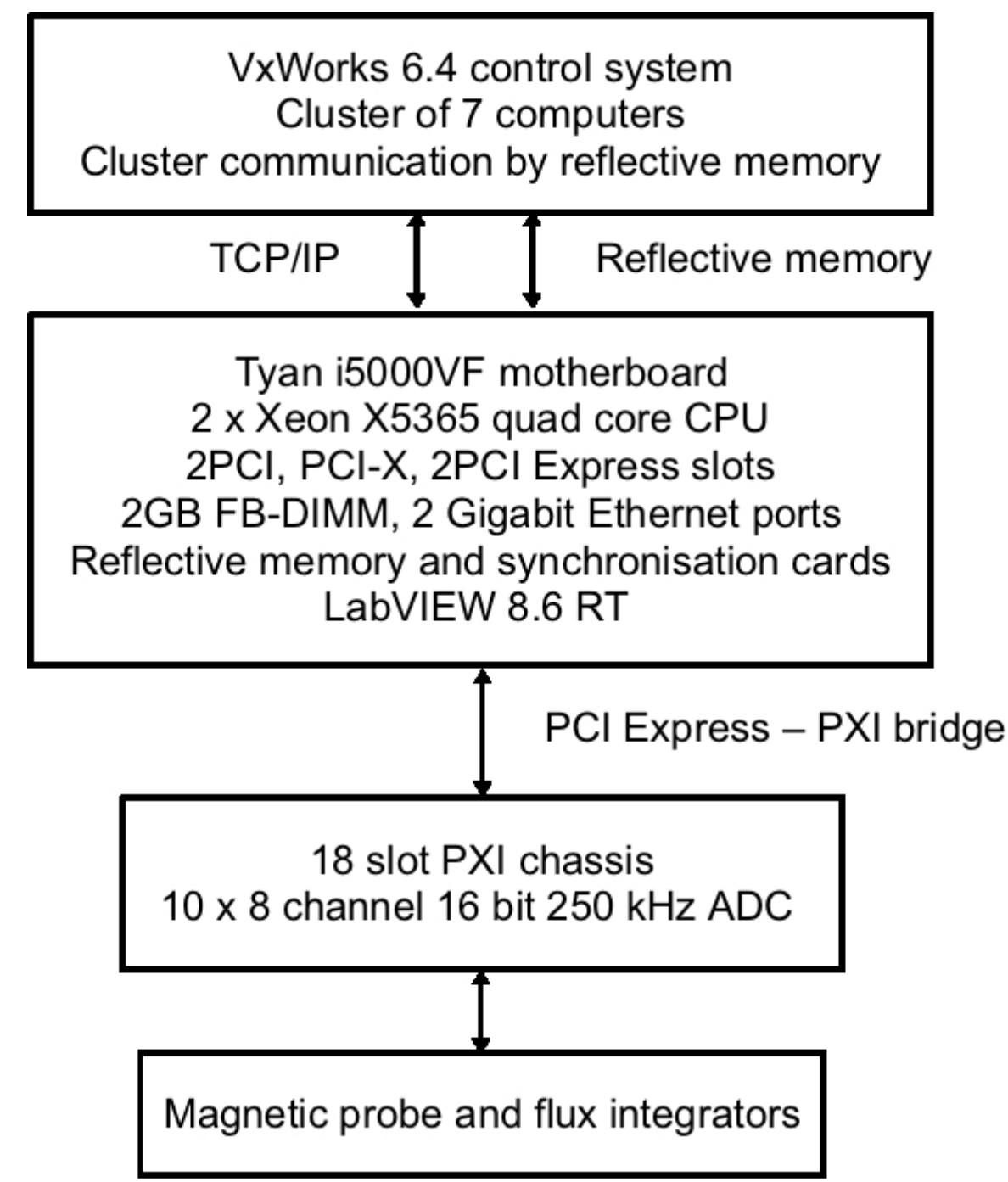

Fig. 1 An overview of the VxWorks control system and new data acquisition system for magnetic probe and flux measurements for real time magnetic flux surfaces.

Real time communication via a central hub to the control system and other diagnostics uses a VMIC 5565PIORC reflective memory card installed in the available PCI-X slot. This unit has a node latency of $500 \mathrm{~ns}$ and achieves a sustained transfer data rate of $170 \mathrm{MB} / \mathrm{s}$ [16]. This reflective memory card is also used for real time communication on KSTAR [10]. Tests using U32 integers indicate that programmed input-output (PIO) speeds of 16 Mbytes/s (write) and $3 \mathrm{Mbytes} / \mathrm{s}$ (read) when sending 8192 bytes could be realised. Tests using U32 integers indicate that DMA speeds of 60 Mbytes/s (write) and 90 Mbytes/s (read) when sending 4096 bytes could be realised. The mean time taken for 2048 single precision real data to be written 
and read using PIO by a sending LabVIEW RT node and receiving Linux node was $550 \mu \mathrm{s}$. A multi-platform diagnostic and control system real time network can be realised using reflective memory .

\section{Calibration}

The measured response of each magnetic probe to the individual poloidal field coil currents in the absence of plasma current is shown in Figure 2. For these calculations, the ASDEX Upgrade coil system is modelled by a set of individual current loops located at a given vertical position and radius. These calculations simply sum the magnetic field and poloidal flux, $\psi$, due to an axisymmetric current loop [17] over each of the individual coils at the given positions of the magnetic probe or the given radius and vertical position of the flux loop. The resulting calculation is reduced to a simple matrix operation requiring $15 \mu \mathrm{s}$. The recorded data of these dedicated calibration pulses are then analysed. The RMS value of the difference between the measured and calculated response in the flat top of the current pulse is minimised by iteratively searching for a self-consistent set of poloidal field coil currents and the magnetic probe or flux loop position, orientation and calibration factor and recalculating the expected response. This reduces systematic errors in the raw signals used for the magnetic equilibrium calculations. A similar procedure was used to commission the newest set of magnetic probes in JET [18].

As shown in Fig. 3, a comparison of the calculated and measured response prior to a tokamak discharge can be used to monitor possible magnetic probe, flux loop or integrator failure in real time. Also, pick up of the toroidal field due to misaligned magnetic probes is corrected in real time. This is particularly important in discharges with a toroidal field ramp. 

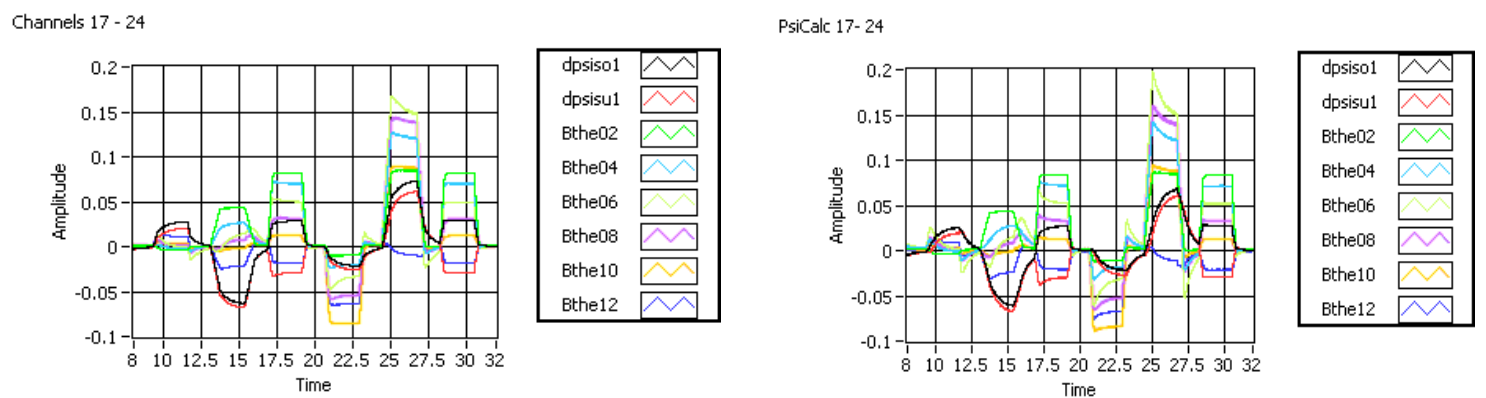

Fig. 2 The measured (left) and calculated (right) magnetic field (Bthenn in [T]) and flux (dpsisnn in $[V s]$ ) response when individual poloidal field coils of the tokamak are pulsed with current. (Individual probes and flux loops are marked by nn)
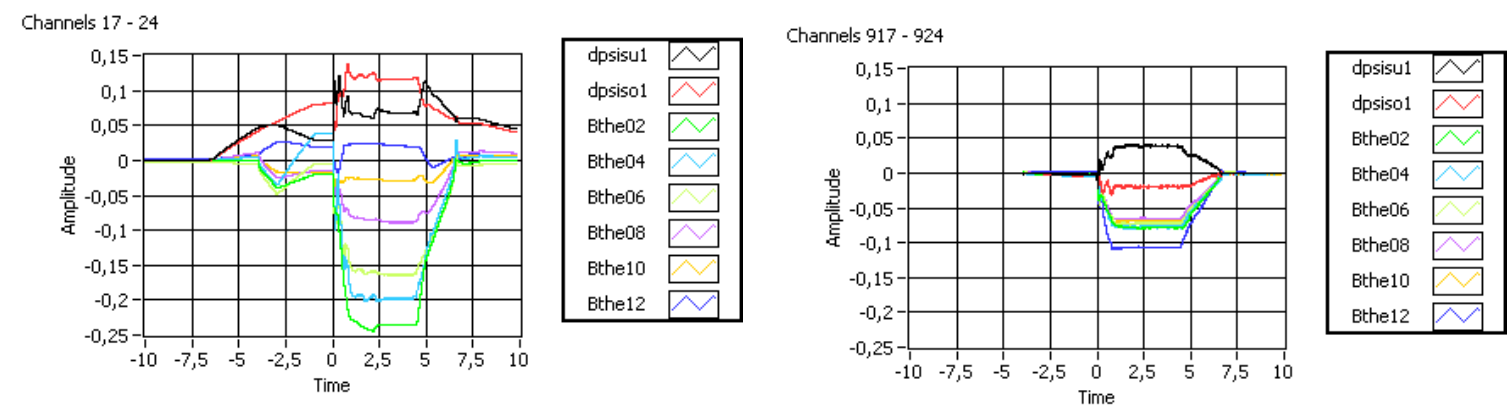

Fig. 3 Magnetic field (Bthenn in [T]) and flux measurements (dpsisnn in [Vs]) during a tokamak discharge (left) and after subtraction of the fields due to poloidal and toroidal field currents (right).(Individual probes and flux loops are marked by nn)

\section{Real time flux surfaces}

The magnetic flux surfaces are required in real time to determine the spatial co-ordinates of the $\mathrm{m} / \mathrm{n}=3 / 2$ or $2 / 1$ rational magnetic flux surface so that the angle of the mirror can be chosen for ECCD power to be deposited on this rational magnetic flux surface. In addition to the magnetic flux surfaces, the electron density profile and microwave beam tracing code calculations, TORBEAM [19], are required in real time. Microwave beam refraction by the electron density gradient needs to be taken into account to steer the mirror to the correct angle for ECCD power deposition on the targeted magnetic flux surface [4].

Function parameterisation reduces the calculation of the magnetic flux surfaces to a matrix multiplication, with $2691 \times 231$ coefficients and a vector of 231 elements. The time taken for this matrix multiplication is reduced by a factor of 4 when parallel processing with the eight 
core system is used. The magnetic flux surfaces are calculated with a cycle time of $1.5 \mathrm{~ms}$. Two dimensional bicubic interpolations of the poloidal flux matrix to find the poloidal flux value at the saddle points and limiter and the determination of the spatial co-ordinates of the contours of the value of poloidal flux corresponding to the required $q=2$ or $q=3 / 2$ rational magnetic flux surface requires a further $1 \mathrm{~ms}$. Including real time plasma current profile information from the MSE diagnostic will impose additional constraints to the equilibrium reconstruction and improve accuracy.

Additionally, the 95 magnetic equilibrium shape and position parameters are calculated with a cycle time of $0.5 \mathrm{~ms}$. This will benefit disruption mitigation experiments in ASDEX Upgrade. In these experiments, the injection of impurity gas was mostly triggered by the locked mode detection system [20]. Two saddle coils mounted in the vessel allow the detection of a locked mode. With the new data acquisition system, the transition above the threshold set for the locked mode trigger to initiate impurity gas injection can be tested with a cycle time smaller than the $1.5 \mathrm{~ms}$ available from the control system of ASDEX Upgrade.

\section{Future real time applications}

Work is in progress to produce a real time version of TORBEAM for LabVIEW RT. The microwave beam tracing equations can be reduced to a coupled system of 19 ordinary differential equations and will profit from parallelisation on a multi-core computer. The Grad-Shafranov equation is the non-linear partial differential equation used to calculate the magnetic equilibrium in a tokamak [21]. The magnetic flux surfaces are calculated after a discharge and are typically on a finer $64 \times 128$ grid. The solution of the Grad-Shafranov equation represents the magnetic equilibrium with the best fit to the experimental data and is more accurate than the functional parameterisation solution as it avoids the compromises 
involved in using a finite number of principal components. Work is in progress to produce a real time LabVIEW RT Grad-Shafranov equation solver. Present real time solvers, such as rtEFIT [22], are based on a cyclic reduction algorithm for the solution of the non-linear partial differential equation [21]. A solution based on a multi-grid algorithm is being investigated [23]. A novel proposal to reformulate the Grad-Shafranov equation and generate an equivalent system that is based on a self-adjoint differential operator is also in development [24]. It is proposed that a cluster of 10 blade servers, with dual quad-core processors in each server, would be an interesting platform to realise this real time application. The ability to calculate magnetic flux surfaces in ASDEX Upgrade from a real time Grad-Shafranov solver would be an alternative way to combine the MSE and magnetic field and flux measurements for magnetic equilibrium fitting.

\section{Summary}

The calculation of magnetic flux surfaces in real time for NTM stabilisation experiments on ASDEX Upgrade with a cycle time of $2.5 \mathrm{~ms}$ has been realised on a multi-core PCI Express system running LabVIEW RT 8.6. The magnetic flux surfaces are required in real time to determine the spatial co-ordinates of the $\mathrm{q}=3 / 2$ or $2 / 1$ rational magnetic flux surface. The angle of the mirror can then be chosen so that ECRH power is deposited on this rational magnetic flux surface. The calculation of plasma position and shape parameters with a cycle time of 0.5 milliseconds is available for disruption mitigation experiments. The desired performance was achieved by taking advantage of parallel matrix operations with the available eight CPU cores. The calculation of magnetic probe and flux loop response to the currents in the poloidal field coils of ASDEX Upgrade with a computation time of $15 \mu$ s will assist in monitoring these signals in real time as magnetic probe, flux loop or integrator failure can be detected prior to a discharge. A real time network based on reflective memory 
for communication between the control system and plasma diagnostics has been brought into operation. 


\section{References}

[1] H.Zohm, G.Gantenbein, A.Gude et. al, Physics of Plasma, 8, 2009, (2001)

[2] P.J. McCarthy, Physics of Plasmas, 6, 3554, (1999)

[3] W.Schneider, P.J.McCarthy, K. Lackner, O. Gruber, K. Behler, P. Martin, R. Merkel, Fusion Eng. Des., 48, 127, (2000)

[4] W.Treutterer, K. Behler, R. Cole, J. Hobirk, M. Jakobi, A. Lohs, K. Lüddecke, G. Neu, G. Raupp, W. Suttrop, D. Zasche, T. Zehetbauer, M. Zilker, ASDEX Upgrade Team, Fusion Eng.Des., 83, 300, (2008)

[5] Data Sheet - ACQ196CPCI 96 channel $250 \mathrm{kHz}$ Simultaneous Digitizer http://www.d-tacq.com/acq196cpci.shtml

[6] Data Sheet - Tyan i5000VF motherboard http://www.tyan.com/product_board_detail.aspx?pid=421

[7] F. Sartori, G. Ambrosino, M. Ariola, A. Cenedese, F. Crisanti, G. De Tommasi, P. Mc Cullen, F. Piccolo, A. Pironti, Other JET-EFDA Contributors, Fusion Eng. Des., 83, 202, (2008)

[8] A. Luchetta, A. Barbalace, G. Manduchi, A. Soppelsa, C. Taliercio, Fusion Eng. Des., 83, 520, (2008)

[9] K. Kurihara, I. Yonekawa, Y. Kawamata, M. Sueoka, H. Hosoyama, S. Sakata, T. Ohshima, M. Sato, K. Kiyono, T. Ozeki, Fusion Eng. Des., 81, 1729, (2006)

[10] H. Jhang and I.S. Choi, Fusion Eng. Des., 73, 35, (2005)

[11] B. Penaflor J.R. Ferron, M.L. Walker, D.A. Humphreys, J.A. Leuer, D.A. Piglowski, R.D. Johnson, B.J. Xiao, S.H. Hahn, D.A. Gates, Fusion Eng. Des., 83, 176, (2008)

[12] B.J.Xiao, D.A. Humphreys, M.L. Walker, A. Hyatt, J.A. Leuer, D. Mueller, B.G. Penaflor, D.A. Pigrowski, R.D. Johnson, A. Welander, Q.P. Yuan, H.Z. Wang, J.R. Luoa, Z.P. Luoa, C.Y. Liu, L.Z. Liu, K. Zhang, Fusion Eng. Des., 83, 181, (2008)

[13] M.Dey and H.D.Pujara, Fusion Engineering Design, 82, 1198, (2007)

[14] L. Giannone et. al, Plasma Physics and Contr. Fusion, 47, 2123, (2005)

[15] M. Mitri et. al, SOFT 2008 Abstract, http://www.ipp.mpg.de/eng/for/veranstaltungen/soft2008/resource/abstracts/253.pdf

[16] Data Sheet - VMIC 5565 Reflective Memory Family http://www.gefanucembedded.com/products/resources/2136

[17] J.D.Jackson, "Classical Electrodynamics", p142, Wiley, (1999)

[18] V. Coccorese, G. Artaserse, G. Chitarin, T. Edlington, S. Gerasimov, S. Peruzzo, 
A. Quercia, C. Sowdenc, JET EFDA contributors, Fusion Eng. Des., 82, 1348, (2007)

[19] E. Poli A.G. Peeters and G.V. Pereverzev,

Computer Physics Communications, 136, 90, (2001)

[20] G. Pautasso, C.J. Fuchs, O. Gruber, C.F. Maggi, M. Maraschek, T. Pütterich, V. Rohde, C. Wittmann, E. Wolfrum, P. Cierpka, M. Beck and the ASDEX Upgrade Team, Nuc. Fusion, 47, 900, (2007)

[21] K. Lackner, Computer Physics Communications, 12, 33, (1976)

[22] J.R. Ferron et al., Proceedings of the 24th European Conference on Controlled Fusion and Plasma Phys Part III, European Physical Society, 1998, 21A (1997) 1125.

[23] A. Vrancic and L. Wenzel, Poster at High Performance Embedded Computing (2008) http://www.1l.mit.edu/HPEC/agendas/proc08/Day3/C7-Vrancic-Poster.pdf

[24] L. Wenzel, National Instruments Corporation, private communication 


\section{Figure Captions}

Figure 1: An overview of the VxWorks control system and new data acquisition system for magnetic probe and flux measurements for real time magnetic flux surfaces.

Figure 2: The measured (left) and calculated (right) magnetic field (Bthenn in [T]) and flux (dpsisnn in $\left[V_{s}\right]$ ) response when individual poloidal field coils of the tokamak are pulsed with current. (Individual probes and flux loops are marked by nn).

Figure 3 : Magnetic field (Bthenn in [T]) and flux measurements (dpsisnn in [Vs]) during a tokamak discharge (left) and after subtraction of the fields due to poloidal and toroidal field currents (right).(Individual probes and flux loops are marked by nn). 